\title{
NEW TYPE OF PERMANENT MAGNET BEAM LINE OPTICS*
}

\author{
S. C. Gottschalk, W. D. Kimura, STI Optronics Inc, Bellevue, WA 94005, USA \\ D. H. Dowell, Boeing Physical Sciences Research Center, Seattle, WA, USA
}

\section{Abstract}

A class of hybrid permanent magnet multipoles that use magnet retraction between ferromagnetic poles to achieve linear, independent field strength adjustments of $40 \%$ and micron level magnetic centerline adjustment are described. Scaling relationships, material choices, passive temperature stabilization, and example designs are discussed.

\section{INTRODUCTION}

Permanent magnet (PM) multipoles are potentially more economical and reliable than DC electromagnets (EM) because of the elimination of high-current power supplies, cooling water and associated plumbing. They are under consideration as an alternative for about half of the 6000 quadrupole magnets in the Next Linear Collider (NLC). A key requirement for those magnets is that they must be magnetically stable with less than $1.4 \mu \mathrm{m}$ of magnetic centerline shift. This is a stringent requirement, which currently available magnets, either EM or PM, cannot easily satisfy.

Another use for PM multipoles is in compact bends, such as the Dundee bend [1], for high power FEL's. At $120 \mathrm{MeV}$ it requires $1.1 \mathrm{~T}, 45 \mathrm{deg}$ dipoles; $1.2 \mathrm{~T}$ quadrupoles (at $5 \mathrm{~cm}$ radius aperture) and $1.0 \mathrm{~T}$ sextupoles. In one part of the bend the edge-to-edge spacing between a quadrupole and a sextupole is only $2.5 \mathrm{~cm}$.

We will describe a 4-magnet quadrupole; see Fig.1, which can be designed to meet both sets of requirement. Field clamps (not shown) are usually included.

The NLC quadrupoles may achieve dynamic centerline adjustments by energizing EM trim coils wrapped aøund pairs of poles or by linearly retracting pairs of magnets FEA shows that for NLC, a 1-micron centerlia adjustment requires $0.12 \mathrm{~W}$. Power scales quadratically with centerline trim so EM adjustments are limited Retraction can achieve arbitrary centerline shifts.

In the compact bend, when the sextupole is very close to the quadrupole there would be substantial field shuntig into a field clamp or significant cross-talk. Using axially directed end magnets and field clamps substantially reduces both problems and allows unrestricte interchanges of the magnets on the beamline.

An important feature of this design is that the strength and centerline tuning are very linear. Standard encoders easily meet the stringent NLC centerline specifications.

This paper only discusses quadrupoles, but radial retraction of magnets has also been applied to dipoles, sextupoles and higher order magnets.

\section{MAGNETIC DESIGN}

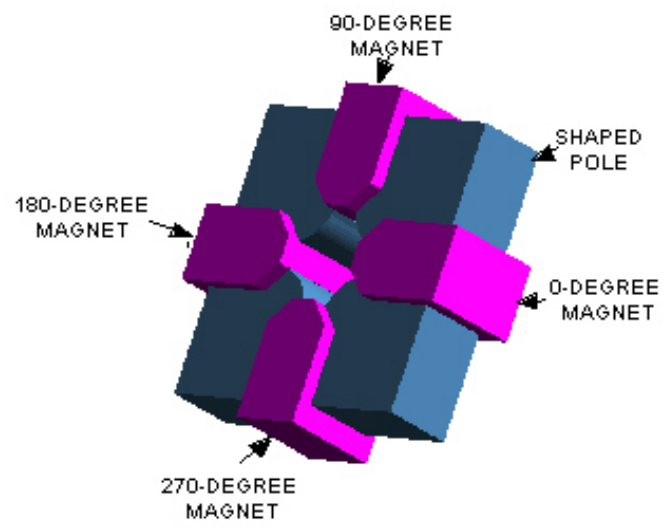

Figure 1: Conceptual design for hybrid PM quadrupole

\subsection{Basic features}

We show a schematic diagram of the adjustable strength quadrupole in Fig. 1 with the retraction structure and motors omitted. Analyses are summarized in Table 1. It is clear that $0.01 \%$ strength adjustments or $1 \mu \mathrm{m}$ centerline shifts can be achieved with off-the shelf encoders. In contrast, adjustable quadrupoles using rotating magnets require ca. $0.01 \mathrm{deg}$ angle resolution.

Table 1: Summary of two PM quadrupole designs

\begin{tabular}{|l|l|l|}
\hline Property & NLC design & $\begin{array}{c}\text { 120 MeV } \\
\text { Dundee bend }\end{array}$ \\
\hline Aperture diameter & $1.3 \mathrm{~cm}$ & $10 \mathrm{~cm}$ \\
\hline Tip field & $0.64-0.96 \mathrm{~T}$ & $1.2 \mathrm{~T}$ \\
\hline Magnet Area & $7 \mathrm{~cm}^{2}$ & $160 \mathrm{~cm}^{2}$ \\
\hline Magnet length & $43 \mathrm{~cm}$ & $14 \mathrm{~cm}$ \\
\hline $\begin{array}{l}\text { Retraction for } 0.01 \% \\
\text { strength adjustment }\end{array}$ & $2 \mu \mathrm{m}$ & $20 \mu \mathrm{m}$ \\
\hline $\begin{array}{l}\text { Magnet shift for } 1 \mu \mathrm{m} \\
\text { centerline adjustment }\end{array}$ & $7 \mu \mathrm{m}$ & $3 \mu \mathrm{m}$ \\
\hline Field clamp & No & Yes \\
\hline End magnets & No & Yes \\
\hline EM trim & Yes & No \\
\hline
\end{tabular}

Magnet transverse and axial overhangs are adjusted to make good use of the material. A magnet recess eliminates third quadrant operation of the magnets. For the NLC design, a $1.3 \mathrm{~mm}$ recess insures none of the magnet operates below $0.2 \mathrm{~B}_{\mathrm{r}}$. For reference purposes, the typical operating condition at the surface of insertion device

* Patent pending 
magnets is about $-0.25 \mathrm{~B}_{\mathrm{r}}$. Each of the poles has an essentially hyperbolic pole tip.

The NLC design is very long so 2D FEA was used, but the poles of the compact bend magnet are $10 \mathrm{~cm}$ long, so 3D FEA was required. All FEA was done with Magnet 6 from Infolytica. Parametric analyses, scripting and solid modeling (ACIS kernel) tools were used for optimizing the magnet and pole shapes and finding scaling relationships.

The field strength adjustment method is shown in Fig. 2. All four magnets can be moved or only a pair. In lower field applications it is reasonable to omit half the magnets to permit side access for diagnostics. The retraction can be accomplished with a variety of mechanical methods. The resulting field strength vs. magnet radial movement is shown in Fig. 3 for NLC quadrupoles. If the magnet needs to be turned off it could be retracted close to an outer shorting plate.

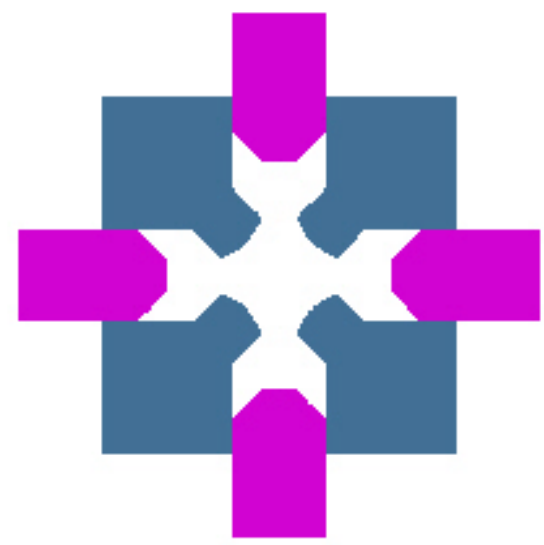

Figure 2: Magnet movement that reduces the field 30\%

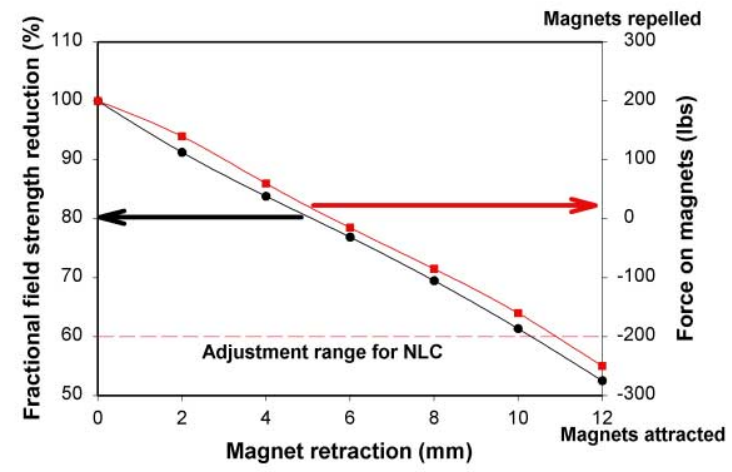

Figure 3: NLC design strength tuning curve. All four magnets are retracted the same amount.

In order to adjust the magnetic centerline, we move two opposing magnets the same amount, $\delta$. For example both the upper and lower magnets would move up to move the magnetic centerline up. This is illustrated in Fig. 4. A 2D FEA analysis relevant to NLC is shown in Fig. 5. Note that the field iso-contours remain linearly spaced and round over most of the beam tube region. This indicates that this does not create any appreciable sextupole. In Fig. $5 \delta$ is $1.5 \mathrm{~mm}$ (half the motion of a single magnet) and the centerline moves by $0.22 \mathrm{~mm}$ or $\delta=7 \mu \mathrm{m}$ for a $1 \mu \mathrm{m}$ magnetic centerline adjustment.

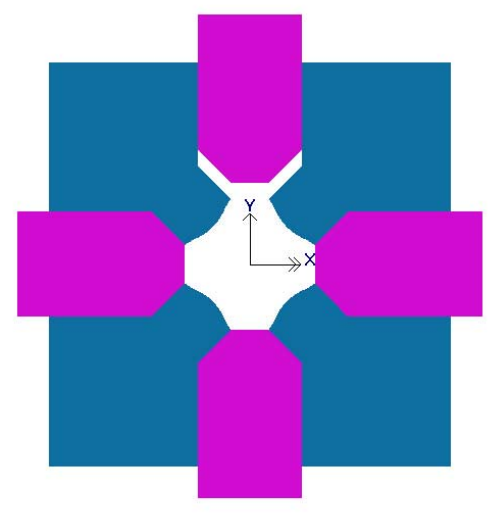

Figure 4: Illustration of vertical centerline adjustment by moving one magnet a different amount. The upper magnet has moved $3.0 \mathrm{~mm}$.

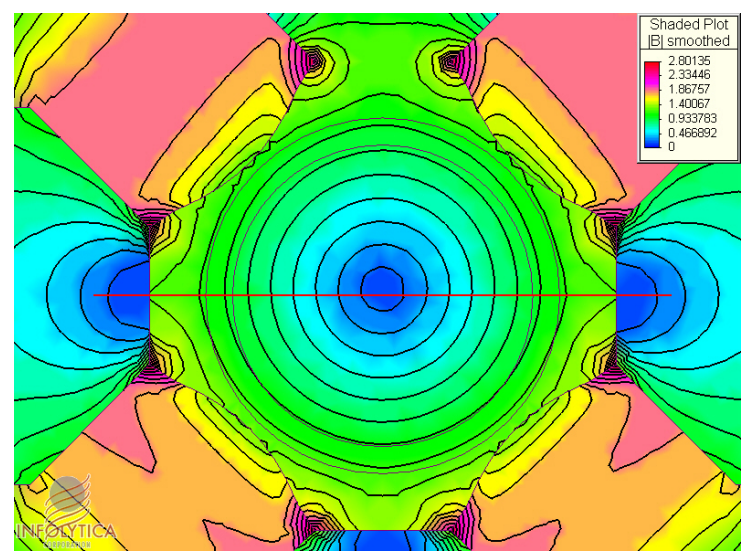

Figure 5: 2D FEA showing movement of magnetic center from vertical motion of one magnet.

A line at $\mathrm{y}=0$ is drawn for reference purposes.

The analysis shown in Fig. 6 indicates that reasonable magnet areas can achieve high pole tip fields. For reference purposes we include EM performance, which is limited by saturation. In the PM design there is essentially no pole saturation, but as usual, adding magnet to regions far away from the gap is not very effective. In any design, adjusting the field strength in and out of saturation will lead to field strength dependent multipoles.

\subsection{Magnetic Material Choices}

The main choices are samarium cobalt $\left(\mathrm{Sm}_{2} \mathrm{Co}_{17}\right)$ and neodymium iron boron $(\mathrm{NdFeB})$. The $\mathrm{Sm}_{2} \mathrm{Co}_{17}$ material is more radiation resistant, but it becomes activated, which can cause a serious handling constraint. It is substantially 
more expensive than $\mathrm{NdFeB}$, has $25 \%$ lower $\mathrm{B}_{\mathrm{r}}$, is extremely brittle and prone to chipping. $\mathrm{NdFeB}$ does not become activated, is strong and hard to chip or break.

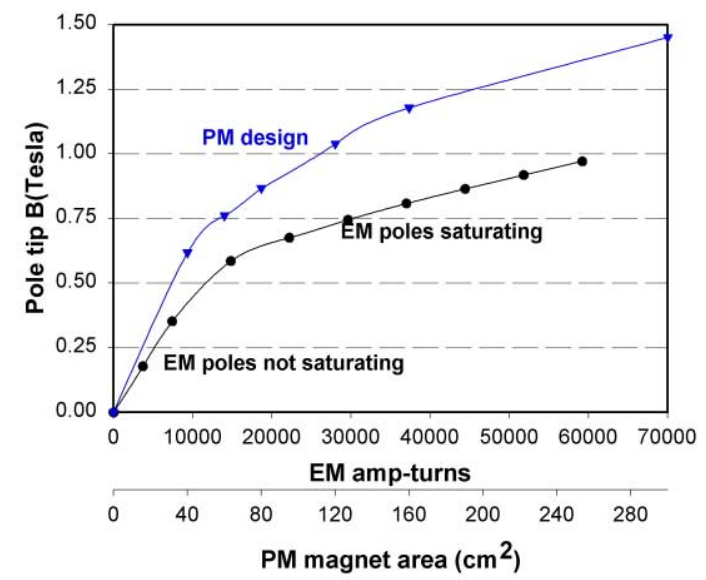

Figure 6: Pole tip fields for hybrid PM designs

While NdFeB can lose strength under irradiation, the ultrahigh coercivity grades show very small remanence losses, $<0.4 \% \pm 0.1 \%$, for absorbed doses up to $3 \mathrm{Mgy}$ from $17 \mathrm{MeV}$ electrons [2]. Essentially no losses were observed under irradiation by ${ }^{60} \mathrm{Co} \gamma$-rays. On the other hand, irradiation by $200 \mathrm{MeV}$ protons does reduce the remanence considerably [3]. Remagnetization fully restored the lost remanence.

Several other ways to reduce the effects of radiation are using lead shielding in the gaps between the vacuum tube and the magnets or using extremely high coercivity $\mathrm{NdFeB}$ or $\mathrm{Sm}_{2} \mathrm{Co}_{17}$ close to the vacuum tube with high coercivity $\mathrm{NdFeB}$ grades further away.

\subsection{Passive Temperature compensation}

The goal of passive temperature compensation is to eliminate known and repeatable thermal effects. One disadvantage of $\mathrm{NdFeB}$ is that $\mathrm{dB} / \mathrm{dt}$ is $-0.1 \%$ /degree which will directly affect the field strength. Using temperature-compensating steels as parallel flux shunts can essentially eliminate this effect. This method has been used for APS NdFeB dipoles [4] to achieve $\Delta B / B \approx$ $2 \times 10^{-5} / \mathrm{deg}-\mathrm{C}$. Fermilab has also studied temperature compensation for low field ferrites [5]. For NLC quads a 9 mm thick flux shunt gives a temperature independent pole tip field that is calculated to be $4 \%$ weaker. Fine-tuning the shunt thickness would also correct field loss due to gap expansion. The compensators are placed in direct thermal contact with the backs of the magnets.

An additional effect is temperature-induced centerline motion. In the simplest example, there are no thermal gradients across the length of the magnet and the base of the magnet is mounted to a thermally stable granite block, but there may be height changes due to thermal expansion/contraction of the frame that holds the magnets, poles and retraction hardware. For NLC the frame might be $10 \mathrm{~cm}$ tall. The height variation might be $2.3 \mu \mathrm{m} / \mathrm{deg}$
$\mathrm{C}$ for aluminum and since the base is granite, centerline variation is half as much or $1.2 \mu \mathrm{m} / \mathrm{deg} C$.

Both of these thermal effects can be corrected by judicious use of temperature-compensating steels having different sizes on the magnets. In the earlier example, as the temperature increases, the centerline will move upwards. In order to move the centerline back down, the bottom magnet needs to be relatively weaker at high temperatures (which is equivalent to retracting the magnet, see Fig. 5) and the top magnet needs to be relatively stronger by the same amount. This is easily achieved by putting less compensator on the top magnet and more compensator on the bottom magnet.

\subsection{Passive force compensation}

The forces on the NLC design are repulsive between $0.96 \mathrm{~T}$ and $0.70 \mathrm{~T}$, and then they are attractive. The zero force region near $0.70 \mathrm{~T}$ could make it difficult to quickly, simply, and accurately position the magnets. A passive approach is to place a stationary back magnet behind the moving magnet. The magnet is oriented in the same direction as the moving magnet. As the moving magnet approaches the back magnet, there would be an additional repulsive force on the moving magnet, thereby achieving the same effect as a spring, but with the added benefit that some magnetic field is generated. In essence this back magnet would replace any springs that might be used to maintain a unidirectional force.

\section{SUMMARY}

A new style of permanent magnet multipole has been described. The design should achieve linear strength and centerline tuning at the micron level by radially retracting the appropriate magnet(s). Magnet position accuracies are modest and should be easily achievable with standard linear encoders. We hope to install a test bend at TJNAF and perform additional tests at the BNL SDL.

\section{ACKNOWLEDGEMENTS}

This work was supported by STI Optronics internal research and development funds and Dept of Interior grant NBCHC010026.

\section{REFERENCES}

[1] D.H. Dowell, in 1997 IEEE Particle Accelerator Conference Proceedings, IEEE Cat. No. 97CH36167, Vol. II, 1888 (1998)

[2] T. Ikeda,et al, Nucl. Inst. Methods Phys. Res. A 407 (1998) 439-442

[3] Y. Ito, et al, "Magnetic flux loss in rare-earth magnets irradiated with $200 \mathrm{MeV}$ protons", unpublished

[4] S. Kim, et al, Proc. 1997 Particle Accleerator Conference, (1998), 3227-3229.

[5] K. Bertsche, J.F. Ostiguy, and W.B. Foster, Proc. 1995 Particle Accelerator Conference (1996) 1381-1383. 